\title{
Predição da massa específica do tomate cereja e do coeficiente de expansão térmica
}

\author{
Prediction of the specific mass of cherry tomatoes and of the coefficient of thermal expansion \\ Predicción de la masa específica del tomate cherry y del coeficiente de expansión térmica
}

Recebido: 27/08/2021 | Revisado: 05/09/2021 | Aceito: 09/09/2021 | Publicado: 12/09/2021

\author{
Ana Clara Melo de Medeiros \\ ORCID: https://orcid.org/0000-0002-7776-0548 \\ Instituto Federal do Rio Grande do Norte, Brasil \\ E-mail: anaclara.melomedeiros@gmail.com \\ Geovana Inácia Cândida Ramalho \\ ORCID: https://orcid.org/0000-0001-7962-389X \\ Instituto Federal do Rio Grande do Norte, Brasil \\ E-mail: geovanaicrmlh@gmail.com \\ Luciana Lorrane Ferreira Linhares \\ ORCID: https://orcid.org/0000-0003-1928-9740 \\ Instituto Federal do Rio Grande do Norte, Brasil \\ E-mail: lulorrane@outlook.com.br \\ Relyson Gabriel Medeiros de Oliveira \\ ORCID: https://orcid.org/0000-0002-2405-0112 \\ Universidade Federal de Campina Grande, Brasil \\ E-mail: relysonrn@gmail.com \\ João Carlos Soares de Melo \\ ORCID: https://orcid.org/0000-0001-9198-8947 \\ Instituto Federal do Rio Grande do Norte, Brasil \\ E-mail: carlos.soares@ifrn.edu.br \\ Joaildo Maia \\ ORCID: https://orcid.org/0000-0002-7671-278X \\ Instituto Federal do Rio Grande do Norte, Brasil \\ E-mail: joaildo.maia@ifrn.edu.br \\ Adair Divino Silva Badaró \\ ORCID: https://orcid.org/0000-0002-1875-7680 \\ Instituto Federal do Rio Grande do Norte, Brasil \\ E-mail: adair.badaro@ifrn.edu.br \\ Carlos Helaidio Chaves da Costa \\ ORCID: https://orcid.org/0000-0002-2066-5152 \\ Universidade Estadual da Paraíba, Brasil \\ E-mail: carlos.chaves@escolar.ifrn.edu.br \\ Flávia Cristina dos Santos Lima \\ ORCID: https://orcid.org/0000-0002-0696-5765 \\ Instituto Federal de Pernambuco, Brasil \\ E-mail: flavia.c.7@hotmail.com
}

\begin{abstract}
Resumo
O objetivo deste trabalho foi obter o melhor modelo matemático para predizer a massa específica da polpa do tomate cereja em função da temperatura $\left(10,20,30,40,50,60\right.$ e $\left.70{ }^{\circ} \mathrm{C}\right)$ e da concentração de sólidos solúveis $(3,0 ; 4,5$ e 6,0 ${ }^{\circ}$ Brix) e, consequentemente, determinar a equação do coeficiente de expansão térmica ( $\beta$ ) para a polpa. As análises da massa específica da polpa do tomate cereja foram realizadas com o uso de picnômetro. A temperatura foi controlada por banho termostático, e a concentração de sólidos solúveis foi determinada com o auxílio do refratômetro portátil. Os modelos matemáticos polinomiais foram ajustados aos dados da massa específica em função da temperatura $\left({ }^{\circ} \mathrm{C}\right)$ e concentração $\left({ }^{\circ}\right.$ Brix). $\mathrm{O}$ coeficiente de expansão térmica $(\beta)$ foi calculado a partir de uma expressão termodinâmica. Os valores médios experimentais da massa específica da polpa de tomate cereja em função das temperaturas e das concentrações de sólidos solúveis variaram de 989,05 a $1032,14 \mathrm{~kg} . \mathrm{m}^{-3}$, tendendo a diminuir com o aumento da temperatura e aumentar com o aumento da concentração de sólidos solúveis. Os modelos matemáticos que melhor se ajustaram aos dados experimentais foram os de $2^{\circ}$ e $3^{\circ}$ graus, com os coeficientes de determinação $\left(R^{2}\right)$ superiores a 0,991. O modelo matemático de $2^{\circ}$ grau foi o escolhido para determinar a equação do coeficiente de expansão térmica, pois facilita a implementação e diminui o tempo de simulação.
\end{abstract}

Palavras-chave: Modelo matemático; Temperatura; Densidade; Concentração.

\begin{abstract}
The objective of this work was to obtain the best mathematical model to predict the specific mass of the cherry tomato pulp depending on the temperature $\left(10,20,30,40,50,60\right.$ and $\left.70{ }^{\circ} \mathrm{C}\right)$ and on the soluble solids' concentration $(3,0 ; 4,5$ and $6,0^{\circ}$ Brix) and, consequently, to determine the thermal expansion coefficient equation $(\beta)$ for the pulp. The
\end{abstract}


specific mass analyses of the cherry tomato pulp were performed with the use of pycnometer. The temperature was controlled through the Thermostatic Bath, and the concentration of soluble solids was determined with the aid portable refractometer. The polynomial mathematical models were adjusted to the specific mass data depending on the temperature $\left({ }^{\circ} \mathrm{C}\right)$ and on the concentration $\left({ }^{\circ} \mathrm{Brix}\right)$. The coefficient of thermal expansion $(\beta)$ was calculated from a thermodynamic expression. The average experimental values of the specific mass of cherry tomato pulp depending on the temperatures and on the soluble solids' concentrations ranged from 989.05 to $1032.14 \mathrm{~kg} . \mathrm{m}^{-3}$, tending to decrease with the increase in temperature and to increase with the increase in the soluble solids concentration. The mathematical models that best fit the experimental data were second and third degree, with the coefficients of determination $\left(\mathrm{R}^{2}\right)$ higher than 0.991 . The mathematical model of 2 nd degree was chosen to determine the coefficient of thermal expansion equation, because it facilitates the implementation and decreases the simulation time.

Keywords: Mathematical model; Temperature; Density; Concentration.

\section{Resumen}

El objetivo de este trabajo fue obtener el mejor modelo matemático para predecir la masa específica de pulpa de tomate cherry en función de la temperatura $\left(10,20,30,40,50,60\right.$ y $\left.70{ }^{\circ} \mathrm{C}\right)$ y la concentración de sólidos solubles $(3,0$; 4,5 y $6,0^{\circ}$ Brix) y, en consecuencia, determinar la ecuación del coeficiente de expansión térmica ( $\beta$ ) para la pulpa. Los análisis de la masa específica de la pulpa de tomate cherry se realizaron con el uso de picnômetro. La temperatura se controló mediante um baño termostático, y la concentración de sólidos solubles se determinó con la ayuda del refractómetro portátil. Los modelos matemáticos polinómicos se ajustaron a los datos de masa específica en función de la temperatura $\left({ }^{\circ} \mathrm{C}\right)$ y la concentración $\left({ }^{\circ}\right.$ Brix). El coeficiente de expansión térmica $(\beta)$ se calculó a partir de una expresión termodinámica. Los valores experimentales medios de la masa específica de pulpa de tomate cherry en función de las temperaturas y las concentraciones de sólidos solubles oscilaron entre 989,05 y $1032,14 \mathrm{~kg} . \mathrm{m}^{-3}$, tendiendo a disminuir con el aumento de la temperatura y a aumentar con el aumento de la concentración de sólidos solubles. Los modelos matemáticos que mejor se ajustaron a los datos experimentales fueron de segundo y tercer grado, con coeficientes de determinación $\left(\mathrm{R}^{2}\right)$ superiores a 0,991 . Se eligió el modelo matemático de $2^{\circ}$ grado para determinar la ecuación del coeficiente de expansión térmica, ya que facilita la implementación y disminuye el tiempo de simulación.

Palabras clave: Modelo matemático; Temperatura; Densidad; Concentración.

\section{Introdução}

O tomate cereja, caracterizado pelo sabor adocicado e tamanho reduzido, é uma das variedades de tomate de maior popularidade a nível mundial. No Brasil, sua produção e comercialização têm sido impulsionadas devido a versatilidade na gastronomia moderna (Lennucci, Cadinu, Taurino, Piro, \& Dalessandro, 2006; Guilherme, Pinho, Cavalcanti, Costa, \& Almeida, 2014). O tomate cereja é caracterizado por nível relativamente alto de antioxidante, vitamina C, fenóis totais e carotenóides, principalmente o licopeno (Lenucci et al. 2006).

Geralmente, o tomate cereja é utilizado in natura em saladas, mas também pode ser utilizado no processamento de polpas, molhos, extratos, entre outros. O consumo do tomate contribui para a melhoria da saúde e qualidade de vida, e, a partir disso, alcança as expectativas do mercado consumidor, que requisita sempre as melhores hortaliças pela sua integridade, aparência, uniformidade, e características nutricionais e sensoriais (Santos, Carmo, \& Abboud, 2016). No entanto, é um alimento altamente perecível, com perdas pós-colheita devido a danos mecânicos, armazenamento, manuseio, transporte inadequado e tempo de exposição no varejo (Mendes, Mendes, Guedes, Silva, \& Arthur, 2020).

O processamento de alimento é uma parte fundamental para as frutas e hortaliças, pois permite prolongar a vida póscolheita, como também, inserir valor comercial, visto que são convertidos em mercadorias com grande importância econômica e demanda, além de possibilitar a geração de emprego para pequenos produtores (Oliveira \& Santos, 2015). O conhecimento de características termofísicas, como a massa específica, difusividade, calor específico, condutividade térmica, se faz necessário no dimensionamento dos equipamentos de processamento de alimentos como evaporadores, bombas, filtros e misturadores, e serve ainda como índice de qualidade do produto final (Pereira, 2013; Bolzan \& Souza, 2007).

O efeito da temperatura e concentrações ( ${ }^{\circ}$ Brix) da massa específica de sucos e polpas de frutas tem sido estudado por diversos pesquisadores. Pereira, Queiroz e Figueirêdo (2002), avaliaram a massa específica de polpa de açaí em três concentrações de sólidos totais $(9,7 ; 12$ e $15,2 \%)$ e diferentes temperaturas $\left(10\right.$ a $\left.50{ }^{\circ} \mathrm{C}\right)$. Bonomo et al. (2009), estudaram o 
comportamento da massa específica do suco de caju em função da temperatura (5 a $\left.80{ }^{\circ} \mathrm{C}\right)$. Guedes, Ramos e Diniz (2010), estudaram o comportamento da massa específica da polpa de melancia em diferentes temperaturas $\left(10,20,30,40,50\right.$ e $\left.60{ }^{\circ} \mathrm{C}\right)$ e concentrações (8, 17, 26 e $35^{\circ}$ Brix). Dantas, Melo, Oliveira, Badaró e Santos (2020), determinaram o comportamento da massa específica da polpa de acerola nas diferentes temperaturas $\left(10\right.$ a $\left.50^{\circ} \mathrm{C}\right)$ e concentrações $\left(8,2 ; 7,2\right.$ e $\left.6,2{ }^{\circ} \mathrm{Brix}\right)$.

Os modelos matemáticos para predição das propriedades termofísicas evoluíram e representam uma oportunidade significativa de melhorar a eficiência de tratamentos térmicos no processamento de alimentos, além, de certa forma, se tornar uma alternativa viável na substituição da determinação experimental destes parâmetros, o qual pode ser muito dispendioso (Egea, Reis, \& Danesi, 2015). Nesse sentido, Zuritz et al. (2005), estudando os modelos matemáticos polinomiais $\left(1^{\circ}, 2^{\circ}\right.$ e $3^{\circ}$ graus) para predizer a massa específica do suco de uva clarificado, verificaram que o modelo polinomial de segundo grau foi o melhor, sendo escolhido para determinar a equação do coeficiente de expansão.

Desse modo, o objetivo do trabalho foi determinar um modelo matemático para descrever o comportamento da massa específica da polpa do tomate cereja em diferentes temperaturas $\left(10\right.$ a $\left.70{ }^{\circ} \mathrm{C}\right)$ e concentrações $\left(3,0 ; 4,5\right.$ e $6,0{ }^{\circ}$ Brix $)$ e obter a equação para o seu coeficiente de expansão térmica.

\section{Metodologia}

Os tomates cerejas foram obtidos no comércio da cidade de Caicó-RN, sendo processados e analisados no laboratório de Química do Campus IFRN/CA. Logo após, foram encaminhados para limpeza em água corrente e mergulhados em solução de hipoclorito de sódio a $50 \mathrm{ppm}$ em um intervalo de 15 minutos. Em seguida, foram enxaguados e despolpados utilizando multiprocessador, envasados e conservados no refrigerador.

A determinação da massa específica da polpa do tomate cereja foi realizada em triplicatas nas temperaturas de 10, 20, 30, 40, 50, 60 e $70^{\circ} \mathrm{C}$ e nas concentrações 3,0; 4,5 e 6,0 ${ }^{\circ}$ Brix. Usou-se pcinômetros antecipadamente calibrados com água destilada e pesados em balança analítica com precisão de $0,0001 \mathrm{~g}$ para cada temperatura escolhida, sendo monitoradas a partir do banho maria (termostático). Os valores da concentração dos sólidos solúveis ( ${ }^{\circ}$ Brix) das polpas foram obtidos através da leitura do refratômetro portátil modelo RT-30ATC, com escala de 0 a $32{ }^{\circ} \mathrm{Brix}$, anteriormente calibrado e regulado a $20{ }^{\circ} \mathrm{C}$ com água destilada.

O cálculo da massa específica foi obtido a partir da Equação 1. Onde, $\rho$ é a massa específica do produto $\left(\mathrm{kg} \cdot \mathrm{m}^{-3}\right)$, v é o volume do picnômetro $\left(\mathrm{m}^{3}\right)$ e m é a massa do produto $(\mathrm{kg})$.

$$
\rho=\mathrm{m} / \mathrm{v}
$$

Os dados referentes à massa específica foram relacionados com a temperatura $\left({ }^{\circ} \mathrm{C}\right)$ e concentração de sólidos solúveis ( ${ }^{\circ}$ Brix), por meio da utilização de uma regressão não linear com polinômios de primeiro, segundo e terceiro graus, de acordo com a análise do modelo matemático e estabelecido conforme a Equação 2. Onde, $\rho$ é a massa específica do produto $\left(\mathrm{kg} . \mathrm{m}^{-3}\right)$, $\mathrm{C}$ é a concentração em sólidos solúveis ( $\left.{ }^{\circ} \mathrm{Brix}\right)$, $\mathrm{T}$ é a temperatura $\left({ }^{\circ} \mathrm{C}\right)$ e $\mathrm{a}_{0}, \mathrm{a}_{1}, \mathrm{a}_{2}, \mathrm{a}_{3}, \mathrm{a}_{4}$, $\mathrm{a}_{5} \mathrm{e} \mathrm{a}_{6}$ são as constantes.

$$
\rho=a_{0}+a_{1} \cdot C+a_{2} \cdot T+a_{3} \cdot C^{2}+a_{4} \cdot T^{2}+a_{5} \cdot C^{3}+a_{6} \cdot T^{3}
$$

A partir da expressão termodinâmica com pressão constante, foi possível obter a equação do coeficiente de expansão térmica, por meio do modelo matemático polinomial que possuiu melhor ajuste para representar a massa específica, relatado por Zuritz et al. (2005). Sendo essa estabelecida pela Equação 3. Onde, $\rho$ é a massa específica do produto $\left(\mathrm{kg} \cdot \mathrm{m}^{-3}\right) ;(\partial \rho / \partial \mathrm{T})_{\mathrm{p}}$ é a derivada parcial da massa específica em função da temperatura a pressão constante. 


$$
\beta=-\left(\frac{1}{\rho}\right)\left(\frac{\partial_{p}}{\partial_{T}}\right)_{p}
$$

Os resultados da massa específicas da polpa de tomate cereja foram submetidos ao delineamento experimento fatorial $3 \times 7$ (três concentrações e sete temperaturas) com três repetições, sendo a comparação entre as médias realizadas pelo teste de Tukey a 5\% de probabilidade, usando-se o programa ASSISTAT, versão 7.7 beta (Silva \& Azevedo, 2016). Foi utilizado o método Quasi-Newton para o ajuste dos modelos matemáticos, através de análises de regressão não linear, com o auxílio do programa Statistica 7.0. Para cada grau de ajuste dos modelos considerou-se a magnitude do coeficiente de determinação $\left(\mathrm{R}^{2}\right)$.

\section{Resultados e Discussão}

Os valores médios experimentais da massa específica da polpa de tomate cereja em função das temperaturas (10 a 70 $\left.{ }^{\circ} \mathrm{C}\right)$ e das concentrações de sólidos solúveis $\left(3,0 ; 4,5\right.$ e $6,0{ }^{\circ}$ Brix), variaram de 989,05 a $1032,14 \mathrm{~kg} . \mathrm{m}^{-3}$. O mesmo comportamento foi obtido por Teixeira, Oliveira e Ramos (2013), estudando a influência da temperatura (10 a $\left.60{ }^{\circ} \mathrm{C}\right)$ e concentração de sólidos solúveis ( 4 a $13^{\circ}$ Brix) nos valores da massa específica da polpa de araçá-boi. Os autores constataram que os valores variaram de $965,79 \mathrm{~kg} \cdot \mathrm{m}^{-3}\left(4^{\circ} \mathrm{Brix}\right.$ e $\left.60^{\circ} \mathrm{C}\right)$ a $1053,06 \mathrm{~kg} \cdot \mathrm{m}^{-3}\left(13^{\circ} \mathrm{Brix} \mathrm{e} 10^{\circ} \mathrm{C}\right)$.

Tabela 1 - Valores médios e desvio padrão da massa específica $\left(\mathrm{kg} \mathrm{m}^{-3}\right)$ da polpa de tomate cereja com diferentes temperaturas e concentrações.

\begin{tabular}{cccc}
\hline Temperatura $\left({ }^{\circ} \mathrm{C}\right)$ & \multicolumn{3}{c}{ Concentração em sólidos solúveis $\left({ }^{\circ}\right.$ Brix $)$} \\
\cline { 2 - 4 } & 3,0 & 4,5 & 6,0 \\
\hline 10 & $1016,10 \pm 0,29^{\mathrm{aC}}$ & $1022,38 \pm 0,84^{\mathrm{aB}}$ & $1032,14 \pm 0,48^{\mathrm{aA}}$ \\
20 & $1011,62 \pm 1,24^{\mathrm{bC}}$ & $1018,13 \pm 0,09^{\mathrm{bB}}$ & $1027,50 \pm 0,39^{\mathrm{bA}}$ \\
30 & $1009,49 \pm 0,38^{\mathrm{bB}}$ & $1010,91 \pm 1,79^{\mathrm{cB}}$ & $1025,74 \pm 0,97 \mathrm{bA}$ \\
40 & $1004,02 \pm 1,49^{\mathrm{cB}}$ & $1005,00 \pm 0,68^{\mathrm{dB}}$ & $1020,08 \pm 1,27 \mathrm{cA}$ \\
50 & $998,60 \pm 1,25^{\mathrm{dB}}$ & $998,71 \pm 0,57^{\mathrm{eB}}$ & $1013,11 \pm 1,29 \mathrm{dA}$ \\
60 & $994,71 \pm 1,35^{\mathrm{deB}}$ & $993,27 \pm 0,50^{\mathrm{fB}}$ & $1008,80 \pm 0,54^{\mathrm{eA}}$ \\
70 & $992,60 \pm 0,24^{\mathrm{eB}}$ & $989,05 \pm 0,63^{\mathrm{gC}}$ & $999,11 \pm 1,29 \mathrm{fA}$ \\
\hline
\end{tabular}

Média geral $=1009,10 \mathrm{~kg} \cdot \mathrm{m}^{-3} ;$ Coeficiente de variação $=0,16 \%$; Ponto médio $=1011,21, \mathrm{DMS}$ colunas $=3,99$ e DMS linhas $=3,13$. A mesma letra minúscula nas colunas e maiúsculas nas linhas não diferem estatisticamente entre si pelo Teste de Tukey ao nível de $5 \%$.

Fonte: Autores (2021).

Nota-se que os valores da massa específica da polpa de tomate cereja tenderam a diminuir estatisticamente com o aumento da temperatura, em todas as concentrações. O mesmo comportamento foi observado por Oliveira e Santos (2020) ao estudarem o comportamento da massa específica da polpa de caju nas temperaturas de 10 a $50{ }^{\circ} \mathrm{C}$ e nas concentrações em sólidos solúveis de 12,4; 0,4 e 8,4 ${ }^{\circ}$ Brix. A redução da massa específica com a temperatura está relacionada com o fenômeno de expansão do fluido, pois a energia fornecida ao sistema aumenta a velocidade de vibração das moléculas, aumentando a distância entre elas (Mercali, Sarkis, Jaeschke, Tessaro, \& Marczak, 2011).

Por outro lado, os valores médios da massa específica da polpa de tomate cereja tenderam a aumentar com o aumento da concentração em sólidos solúveis ( ${ }^{\circ} \mathrm{Brix}$ ), nas diferentes temperaturas, exceto na temperatura de $70{ }^{\circ} \mathrm{C}$. Dantas et al. (2020) ao estudarem a influência sofrida pela massa específica da polpa de acerola submetida a diferentes temperaturas (10 a $50^{\circ} \mathrm{C}$ ) e concentrações de sólidos solúveis (8,2; 7,2 e 6,2 ${ }^{\circ}$ Brix), observaram o aumento dos valores da massa especifica a partir do aumento da concentração de sólidos solúveis da polpa de acerola, nas diferentes temperaturas. De acordo com Chin, 
Chan, Yusof, Chuah e Talib (2008), este aumento deve-se à remoção de água dos constituintes (proteínas, carboidratos e sólidos solúveis totais) da polpa mais concentrada.

De acordo com os dados obtidos, os modelos que melhor se ajustaram aos dados experimentais foram os polinomiais de $2^{\circ}$ e $3^{\circ}$ grau, com os coeficientes de determinação $\left(\mathrm{R}^{2}\right)$ superiores a 0,991 (Tabela 2). Porém, o modelo matemático descrito pelo polinômio de $1^{\circ}$ grau também pode ser utilizado, pois apresentou $\mathrm{R}^{2}>0,96$, sendo considerando um bom ajuste.

Tabela 2 - Parâmetros dos modelos matemáticos polinomiais com seus respectivos coeficientes de determinação da massa específica da polpa do tomate cereja.

\begin{tabular}{cccc}
\hline Coeficientes & Polinômio de $1^{\circ}$ grau & Polinômio de $2^{\circ}$ grau & Polinômio de $3^{\circ}$ grau \\
\hline $\mathrm{a}_{0}$ & 1007,733 & 1049,785 & 364,1294 \\
$\mathrm{a}_{1}$ & 4,849668 & $-16,4813$ & 477,7774 \\
$\mathrm{a}_{2}$ & $-0,516415$ & $-0,357250$ & $-0,209530$ \\
$\mathrm{a}_{3}$ & - & 2,370107 & $-111,690$ \\
$\mathrm{a}_{4}$ & - & $-0,001990$ & $-0,006313$ \\
$\mathrm{a}_{5}$ & - & - & 8,448867 \\
$\mathrm{a}_{6}$ & - & - & 0,000036 \\
$\mathrm{R}^{2}$ & 0,9688 & 0,9917 & 0,9919 \\
\hline
\end{tabular}

Fonte: Autores (2021).

Dantas et al. (2020) observaram que o modelo matemático que melhor se ajustou aos dados experimentais da massa específica da polpa de acerola foi o de $3^{\circ}$ grau, com o coeficiente de determinação $\mathrm{R}^{2}>0,99$. Entretanto, os autores consideram que os demais modelos polinomiais de $1^{\circ}$ e $2^{\circ}$ graus também apresentaram resultados satisfatórios com $\mathrm{R}^{2}$ iguais a 0,96 e 0,99 , respectivamente. Sani, Hedayati e Arianfar (2014), estudando o modelo matemático mais adequado para descrever o comportamento do suco de melão, observaram que o modelo polinomial resultante da combinação entre a concentração linear e temperatura quadrática foi aquele que melhor predizia a massa específica do suco de melão, pois obtiveram o coeficiente de determinação igual a 0,999 .

Tem-se na Figura 1, a superfície de resposta para o modelo matemático de $2^{\circ}$ grau, que descreveu o comportamento da massa específica da polpa de tomate cereja em função da temperatura e da concentração, de acordo o modelo polinomial de $2^{\circ}$ grau apresentado na Tabela 2. Giraldo, Cruz e Sanabria (2017) escolheram o mesmo modelo ( $2^{\circ}$ grau) para gerar a superfície de resposta, pois observaram que os coeficientes quadráticos de temperatura $\left(\mathrm{T}^{2}\right)$ e concentração de $\left(\mathrm{C}^{2}\right)$ do modelo combinado apresentaram efeito significativo, com nível de confiança de 95\%, na massa específica do suco clarificado de groselha, com coeficiente de determinação igual a 0,994. 
Figura 1 - Massa específica da polpa de tomate cereja em função da concentração e temperatura.

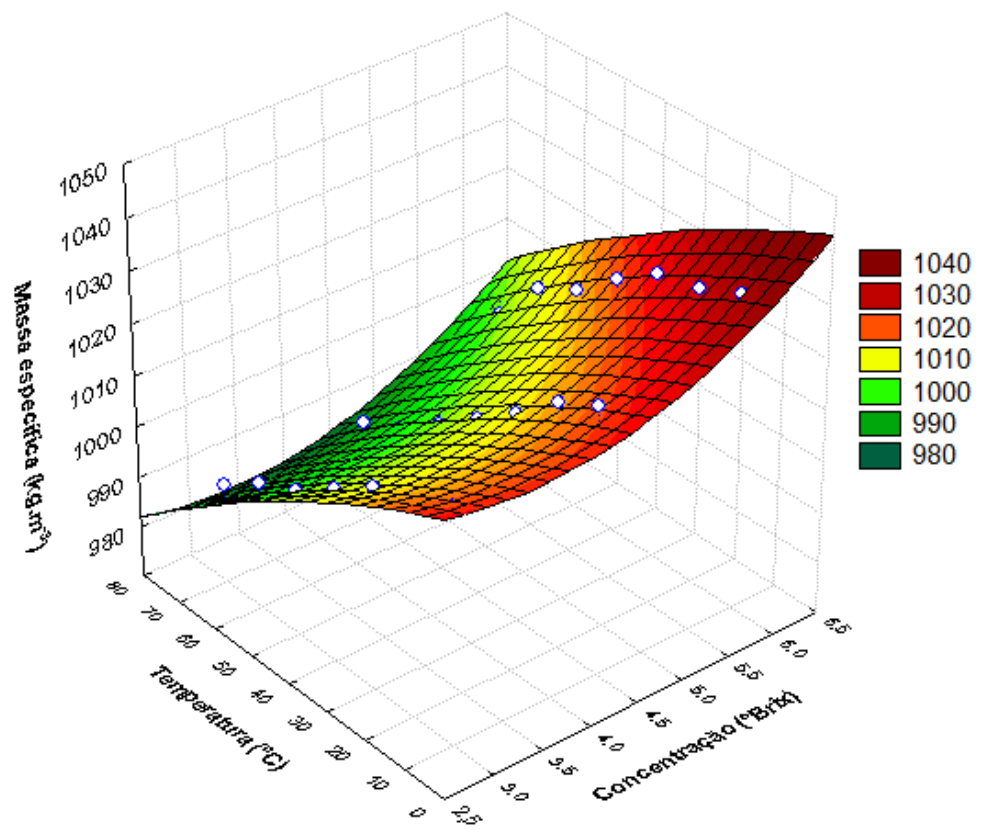

Fonte: Autores (2021).

Portanto, o modelo matemático escolhido para obtenção do coeficiente de expansão térmica ( $\beta$ ) da polpa de tomate cereja foi o polinômio de segundo grau, já que o coeficiente de determinação do modelo de segundo grau $\left(\mathrm{R}^{2}=0,9917\right)$ foi inferior apenas na quarta casa após a vírgula do modelo de terceiro grau $\left(\mathrm{R}^{2}=0,9919\right)$, facilitando a sua implementação e diminuindo o tempo de simulação. Sendo assim, o coeficiente de expansão térmica ( $\beta$ ) obtido para a polpa de tomate cereja foi representada pela equação 4. Resultado semelhante foi encontrado por Zuritz et al. (2005), ao estudarem os modelos polinomiais mais adequados para determinar a massa especifica do suco de uva clarificado e a equação do coeficiente de expansão térmica, no qual os autores também utilizaram o polinômio de $2^{\circ}$ grau.

$$
\beta=\frac{0,357250+0,003980 \cdot \mathrm{T}}{1049,785-16,4813 \cdot \mathrm{C}-0,357250 \cdot \mathrm{T}+2,370107 \cdot \mathrm{C}^{2}-0,001990 \cdot \mathrm{T}^{2}}
$$

\section{Conclusão}

Os valores médios experimentais da massa específica da polpa de tomate cereja em função das temperaturas e das concentrações de sólidos solúveis variaram de 989,05 a 1032,14 kg . $\mathrm{m}^{-3}$, tendendo a diminuir com o aumento da temperatura e aumentar com o aumento da concentração de sólidos solúveis. Os modelos matemáticos que melhor se ajustaram aos dados experimentais foram os de $2^{\circ}$ e $3^{\circ}$ graus, com os coeficientes de determinação $\left(\mathrm{R}^{2}\right)$ superiores a 0,991 . O modelo matemático escolhido para obter a equação do coeficiente de expansão térmica foi o modelo polinomial de $2^{\circ}$ grau por facilitar a implementação e diminuir o tempo de simulação.

\section{Agradecimentos}

Ao Instituto Federal do Rio Grande do Norte, Campus Caicó, e ao CNPq pelo incentivo financeiro concebido para o desenvolvimento dessa pesquisa. 


\section{Referências}

Bolzan, T. G., \& Souza, D. D. (2007). Estudo do comportamento da massa específica de suco de laranja em função da temperatura e da concentração. Salão de Iniciação Científica (19.: 2007: Porto Alegre, RS). Livro de resumos. UFRGS, 2007.

Bonomo, R. C. F., Fontan, R., de Souza, T. S., Veloso, C. M., Reis, M. F. T., \& Souza, S. D. (2009). Thermophysical properties of cashew juice at different concentrations and temperatures. Revista Brasileira de Produtos Agroindustriais, 11(1), 35-42. doi: http://dx.doi.org/10.15871/1517-8595/rbpa.v11n1p35-42

Chin, N. L., Chan, S. M., Yusof, Y. A., Chuah, T. G., \& Talib, R. A. (2008). Prediction of physicochemical properties of pummelo juice concentrates as a function of temperature and concentration. International Journal of Food Engineering, 4(7). doi: https://doi.org/10.2202/1556-3758.1529

Dantas, H. C., Melo, J. C. S., Oliveira, R. G. M., Badaró, A. D. S., \& Santos, E. R. M. (2020). Predição da massa específica da polpa de acerola a partir de modelos matemáticos. Revista Brasileira de Gestão Ambiental, 14(1), 12-16. <https://www.gvaa.com.br/revista/index.php/RBGA/article/view/7633/7180>

Egea, M. B., Reis, M. H. M., \& Danesi, E. D. G. (2015). Aplicação de modelos matemáticos preditivos para o cálculo das propriedades termofísicas do palmito pupunha. Revista Brasileira de Produtos Agroindustriais, Campina Grande, 17(2), 179-187. doi: http://dx.doi.org/10.15871/15178595/rbpa.v17n2p179-187

Giraldo, G. I., Cruz, C. D., \& Sanabria, N. R. (2017). Propiedades físicas del jugo de uchuva (Physalis peruviana) clarificado en función de la concentración y la temperatura. Información tecnológica, 28(1), 133-142. doi: http://dx.doi.org/10.4067/S0718-07642017000100013

Guedes, D. B., Ramos, A. M., \& Diniz, M. D. M. S. (2010). Efeito da temperatura e da concentração nas propriedades físicas da polpa de melancia. Brazilian Journal of Food Technology, 13(4), 279-285. doi: http://dx.doi.org/10.4260/BJFT2010130400037

Guilherme, D. D. O., de Pinho, L., Cavalcanti, T. F. M., da Costa, C. A., \& de Almeida, A. C. (2014). Análise sensorial e físico-química de frutos tomate cereja orgânicos. Revista Caatinga, 27(1), 181-186. Disponível em: <http://www.redalyc.org/articulo.oa?id=237130153022>.

Lenucci, M. S., Cadinu, D., Taurino, M., Piro, G., \& Dalessandro, G. (2006). Antioxidant composition in cherry and high-pigment tomato cultivars. Journal of agricultural and food chemistry, 54(7), 2606-2613. doi: https://doi.org/10.1021/jf052920c

Mendes, K. F., Mendes, K. F., Guedes, S. F., Silva, L. C. A. S., \& Arthur, V. (2020). Evaluation of physicochemical characteristics in cherry tomatoes irradiated with 60Co gamma-rays on post-harvest conservation. Radiation Physics and Chemistry, 177, 109139. doi: https://doi.org/10.1016/j.radphyschem.2020.109139

Mercali, G. D., Sarkis, J. R., Jaeschke, D. P., Tessaro, I. C., \& Marczak, L. D. F. (2011). Physical properties of acerola and blueberry pulps. Journal of Food Engineering, 106(4), 283-289. doi: https://doi.org/10.1016/j.jfoodeng.2011.05.010

Oliveira, E. N. A. D., \& Santos, D. D. C. (2015). Tecnologia e processamento de frutos e hortaliças.

Oliveira, R. G. M., Melo, J. C. S., Badaró, A. D. S., da Costa, C. H. C., Dantas, H. C., Dantas, E. H., \& dos Santos Lima, F. C. (2020). Correlação matemática da massa específica da polpa de caju em diferentes temperaturas e concentrações. Brazilian Journal of Development, 6(5), $27844-27849$. doi: https://doi.org/10.34117/bjdv6n5-290

Pereira, C. G. (2013). Propriedades termofísicas e comportamento reológico de polpa de acerola em diferentes concentrações e temperaturas. Dissertação (mestrado), Universidade Federal de Lavras, Lavras, MG, Brasil. http://repositorio.ufla.br/bitstream/1/751/1/DISSERTACAO_ Propriedades $\% 20$ termof\%C3\%ADsicas\%20e\%20comportamento\%20reol\%C3\%B3gico....pdf

Pereira, E. A., Queiroz, A. J. D. M., \& de Figueirêdo, R. M. (2002). Massa específica de polpa de açaí em função do teor de sólidos totais e da temperatura. Revista Brasileira de engenharia agrícola e ambiental, 6, 526-530. doi: https://doi.org/10.1590/S1415-43662002000300025

Sani, A. M., Hedayati, G., \& Arianfar, A. (2014). Effect of temperature and concentration on density and rheological properties of melon (Cucumis melo L. var. Inodorus) juice. Nutrition \& Food Science., 0034-6659. doi: https://doi.org/10.1108/NFS-06-2013-0065

Santos, C. A., Carmo, M. G. F, Abboud, A. C. S. (2016). Novo nicho tomate cereja orgânico. Revista Campo \& Negócios., 137, 16-20. https://www.researchgate.net/publication/340875557_Novo_nicho_tomate_cereja_organico.

Silva, F. D. A. S., \& Azevedo, C. A. V. de (2016). The Assistat Software Version 7.7 and its use in the analysis of experimental data. African Journal of Agricultural Research, 11(39), 3733-3740. doi: https://doi.org/10.5897/AJAR2016.11522

Teixeira, T. R., Oliveira, A. N., \& Ramos, A. M. (2013). Efeitos da temperatura e concentração nas propriedades físicas da polpa de araçá-boi. Boletim do Centro de Pesquisa de Processamento de Alimentos, 31(2). 07-10 doi: http://dx.doi.org/10.5380/cep.v31i2.34957

Zuritz, C. A., Puntes, E. M., Mathey, H. H., Pérez, E. H., Gascón, A., Rubio, L. A., \& Cabeza, M. S. (2005). Density, viscosity and coefficient of thermal expansion of clear grape juice at different soluble solid concentrations and temperatures. Journal of Food Engineering, 71(2), 143-149. doi: https://doi.org/10.1016/j.jfoodeng.2004.10.026 